\title{
Preoperative Platelet to Albumin Ratio Predicts Outcome of Patients with Cholangiocarcinoma
}

\author{
NOBUHIRO SAITO ${ }^{1,2}$, YOSHIHIRO SHIRAI $^{1,2}$, TAKASHI HORIUCHI $^{1,2}$, HIROSHI SUGANO $^{1,2}$, \\ HIROAKI SHIBA $^{1}$, TARO SAKAMOTO ${ }^{1}$, TADASHI UWAGAWA ${ }^{1,3}$ and KATSUHIKO YANAGA ${ }^{1}$ \\ ${ }^{1}$ Department of Surgery, ${ }^{2}$ Division of Gene Therapy, Research Center for Medical Sciences, and \\ ${ }^{3}$ Division of Medical Oncology and Hematology, Department of Internal Medicine, \\ The Jikei University School of Medicine, Tokyo, Japan
}

\begin{abstract}
Background: The purpose of this study was to evaluate the prognostic index of the preoperative platelet to albumin ratio (PAR) in patients who underwent primary resection for cholangiocarcinoma. Patients and Methods: A total of 59 patients were divided into two groups: those with $P A R \geq 72.6 \times 10^{3}$ or $<72.6 \times 10^{3}$ according to the area under the receiver operating characteristics curve. Results: PAR was significantly inversely associated with overall $(O S)$ and diseasefree (DFS) survival on univariate analysis. PAR showed significance on multivariate analysis for OS (hazard ratio $=6.232$, 95\% confidence interval $=1.283-30.279, p=0.023$ ), along with tumor differentiation $(p=0.009)$, nodal involvement $(p=0.001)$, intraoperative blood loss $(p=0.001)$, and serum carcinoembryonic antigen $(C E A)(p=0.012)$. High PAR was also significantly associated poor DFS on multivariate analysis (hazard ratio $(H R)=4.422,95 \%$ confidence interval $(C I)=1.168$ $16.732, p=0.029)$, along with tumor differentiation $(p=0.009)$. Conclusion: PAR is a useful prognostic index for $O S$ and DFS in patients with cholangiocarcinoma after primary resection. By accumulating cases prospectively, this new index may be a reference for use before neoadjuvant chemotherapy.
\end{abstract}

Cholangiocarcinoma is characterized by tumor aggressiveness with limited choices of curative treatment (1); it has an extremely poor prognosis with a high mortality rate all over the world. Most cases are found to be unresectable as a result of cancer progression. The median survival time (MST) of patients with unresectable cholangiocarcinoma is only 3-6 months (2). In resectable cases, the 5-year overall survival for those with

Correspondence to: Nobuhiro Saito, MD, The Jikei University School of Medicine, 3-25-8, Nishi-shinbashi, Minato-ku, Tokyo 105-8461, Japan. Tel: +81 334331111, ext. 3401, Fax: +81 334331230,e-mail: h24dr-saito@jikei.ac.jp

Key Words: Cholangiocarcinoma, bile duct neoplasm, prognosis, surgery. stage 3 cholangiocarcinoma is only $10 \%$ (3). Even for marginnegative resection cases, the MST after curative resection is reported to be 46 months (4). Recently, several studies for cholangiocarcinoma, including neoadjuvant or adjuvant chemotherapy, have been published $(5,6)$. To select an effective therapeutic strategy, accurate preoperative estimation of the prognosis is important. Some reports have emphasized the importance of preoperative platelet count and postoperative prognosis, including cancer-specific mortality $(7,8)$. Moreover, other reports imply that a low serum albumin level is associated with a poor postoperative outcome $(9,10)$. Therefore, in order to make an index reflecting both these factors, we analyzed the relation between the platelet to albumin ratio (PAR) and postoperative prognosis. To our best knowledge, this is the first study that has analyzed the significance of PAR for cholangiocarcinoma. In the current study, we evaluated the correlation between long-term postoperative outcomes of the patients with cholangio-carcinoma and PAR.

\section{Patients and Methods}

Between 2010 and 2014, 60 patients with primary cholangiocarcinoma underwent primary tumor resection. Although this seems a small sample size, this is because of the rarity of this disease and short-term observation due to its poor prognosis.

Depending on tumor localization, we performed hepatic resection for perihilar cholangiocarcimona, and pancreaticoduodenectomy for distal bile duct cancer at Jikei University Hospital, Tokyo, Japan. Of these, there was no case for which hepato-pancreatico-duodenectomy was performed. We conducted a retrospective review of a maintained database of patients who were histologically diagnosed with primary cholangiocarcinoma. Of these, one patient was excluded as having cholangitis after postoperative pathological diagnosis, leaving the remaining 59 cases for this study. This study was approved by the Ethics Committee of Jikei University School of Medicine (approval number: 27-177 (8062)). Our Ethics Committee conforms to the provisions of the Declaration of Helsinki. The detailed pathological factors were based on the seventh edition of the Union for International Cancer Control TNM Classification (11). All excised specimens were diagnosed as adenocarcinoma at the Department of Pathology, Jikei University Hospital, Tokyo, Japan. The laboratory 
data were collected shortly before the operation was determined. All patients underwent operation without neoadjuvant chemotherapy.

We investigated the relation between clinicopathological variables and overall (OS) and disease-free (DFS) survival after curative primary resection by univariate and multivariate analyses. Glasgow Prognostic Score (GPS) was determined as previously described (12). The neutrophil to lymphocyte ratio (NLR) (13), platelet to lymphocyte ratio (PLR) (14), prognostic nutrition index (PNI) (15), and $\mathrm{C}$-reactive protein to albumin ratio (CAR) (16) were calculated on the basis of previous studies. PAR was defined as the platelet count divided by serum albumin level as we previously reported for pancreatic cancer (17). The cut-off values of these inflammationbased prognostic scores were classified into two groups for the logrank test and Cox proportional regression model based on receiver operating characteristic (ROC) curve analysis using 3-year survival Recurrence of cholangiocarcinoma was defined as a local or distant metastatic tumor that was newly found by ultrasonography, computed tomography, or magnetic resonance imaging with or without an increase in serum carcinoembryonic antigen (CEA).

We analyzed the relationship between clinicopathological variables including inflammation-based prognostic scores and survival after hepatic or pancreatic resection for cholangiocarcinoma by univariate and multivariate analysis of the following 16 factors: tumor stage, differentiation, size, nodal involvement, resection margin status, preoperative biliary drainage, localization of tumor, operative time, intraoperative blood loss, serum CEA, GPS, NLR, PLR, PNI, CAR, and PAR. We also evaluated the relationship between PAR and clinicopathological variables by univariate analysis. In the current study, carbohydrate antigen 19-9 (CA19-9) was not included. Although the serum CA19-9 level was useful for diagnosis or follow-up on recurrence, the level of CA19-9 did not necessarily reflect the prognosis in this study.

For both perihilar and distal cholangiocarcinoma, liver function was Child-Pugh classification A or B.

Statistical analysis. Continuous variables are expressed as the median and interquartile range. Categorical variables are expressed as absolute numbers. Univariate analyses for categorical data were performed using chi-square tests or Fisher's exact test. The area under the ROC curve (AUC) was calculated to determine the cutoff value for each prognostic score. The OS and DFS rates were determined by the Kaplan-Meier method, using Graphpad PRISM ver. 6.07 (GraphPad Software, Inc., La Jolla, CA, USA). Comparisons of OS and DFS were performed using the log-rank test for univariate analysis and the Cox proportional regression model with backward elimination stepwise approach for multivariate analysis, using SPSS 23.0 (IBM Corp., Armonk, NY, USA). $p$ Values were considered statistically significant when less than 0.05 .

\section{Results}

Patient characteristics. Table I lists patient characteristics. Among the study population, the median age was 69 years ( 25 to 75 percentile of 62.5-73.5 years), and 42 patients were male. A majority, $67.8 \%$ of patients, had stage II or III cholangiocarcinoma. Median OS and DFS after surgery for cholangiocarcinoma with curative intent were 22.8 and 18 months, respectively, and 3-year survival rates were $49.3 \%$ and $32.3 \%$, respectively.
Table I. Patient characteristics $(n=59)$.

\begin{tabular}{lcc}
\hline Factor & Median or number & Range (IQR) \\
\hline Age (years) & 69 & $62.5-73.5$ \\
Gender (male:female) & $42: 17$ & \\
Disease-free survival (years) & 1.5 & $0.7-2.0^{*}$ \\
Overall survival (years) & 1.9 & $1.3-2.9^{*}$ \\
White blood cell count $\left(\times 10^{3} / \mathrm{ml}\right)$ & 6.6 & $5.0-7.9$ \\
Neutrophil count $\left(\times 10^{3} / \mathrm{ml}\right)$ & 4.1 & $2.9-5.0$ \\
Lymphocyte count $\left(\times 10^{3} / \mathrm{ml}\right)$ & 1.5 & $1.3-2.0$ \\
Platelet count $\left(\times 10^{3} / \mathrm{ml}\right)$ & 220 & $188-274$ \\
Serum albumin $(\mathrm{g} / \mathrm{dl})$ & 3.9 & $3.4-4.2$ \\
Serum CRP $(\mathrm{mg} / \mathrm{l})$ & 0.2 & $0.1-0.5$ \\
Serum CEA $(/ \mathrm{ml})$ & 46.0 & $19.5-106.0$ \\
PLR & 149.4 & $106.2-191.8$ \\
NLR & 2.4 & $1.7-3.8$ \\
PNI & 46.0 & $41.3-49.8$ \\
CAR & 0.05 & $0.02-0.13$ \\
PAR $\left(\times 10^{3}\right)$ & 60.6 & $44.3-74.5$ \\
\hline
\end{tabular}

IQR: Interquartile range; CEA: carcinoembryonic antigen; PLR: platelet to lymphocyte ratio; NLR: neutrophil to lymphocyte ratio; GPS: Glasgow prognostic score; PNI: prognostic nutritional index; CAR: CRP to albumin ratio; PAR: platelet to albumin ratio; $* 95 \%$ confidence interval.

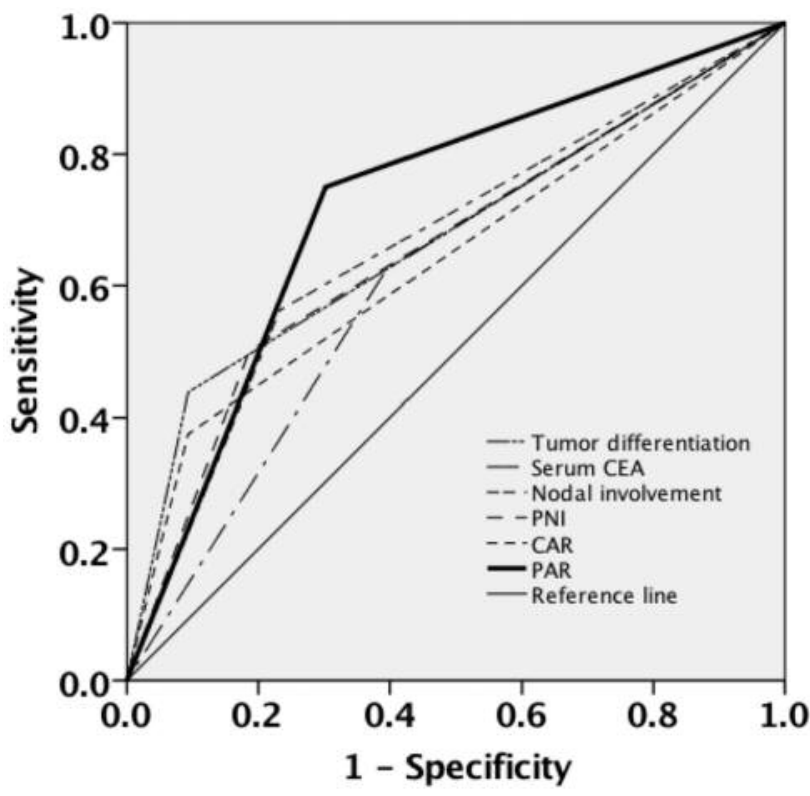

Figure 1. The receiver operating characteristics (ROC) analysis of study factors. The platelet to albumin ratio (PAR) had the highest area under the curve value $(0.709 \pm 0.08, p=0.002)$ compared to other prognostic scores as independent risk factors.

Relationship between clinical variables and OS. The relationship between clinicopathological variables, including inflammation-based prognostic scores and OS, are shown in Table II. In univariate analysis, significant prognostic factors 
Table II. Univariate and multivariate analysis of clinicopathological variables in relation to overall survival.

\begin{tabular}{|c|c|c|c|c|c|c|}
\hline \multirow[b]{2}{*}{ Factor } & \multirow[b]{2}{*}{ Subgroup } & \multirow[b]{2}{*}{$\mathrm{N}$} & \multicolumn{2}{|c|}{ Univariate analysis } & \multicolumn{2}{|c|}{ Multivariate analysis } \\
\hline & & & HR $(95 \% \mathrm{CI})$ & $p$-Value & HR $(95 \% \mathrm{CI})$ & $p$-Value \\
\hline Tumor stage & II, III/I & $40 / 19$ & $2.788(1.057-7.353)$ & 0.038 & & n.s \\
\hline Tumor size (mm) & $\geq 30.5 /<30.5 \mathrm{~mm}$ & $41 / 18$ & $1.878(0.652-5.410)$ & 0.242 & & n.s \\
\hline \multirow[t]{3}{*}{ Tumor differentiation } & Poor & 11 & $10.06(2.394-42.26)$ & 0.002 & $3.238(1.349-7.771)$ & 0.009 \\
\hline & Moderate & 30 & $1.596(0.452-5.635)$ & & & \\
\hline & Well (ref) & 18 & & & & \\
\hline Nodal involvement & Positive/negative & $19 / 40$ & $3.498(1.134-10.79)$ & 0.029 & & 0.001 \\
\hline Resection margin status & Positive/negative & $13 / 46$ & $1.535(0.485-4.856)$ & 0.466 & & n.s \\
\hline Preoperative biliary drainage & Positive/negative & $16 / 43$ & $1.218(0.410-3.621)$ & 0.723 & & n.s \\
\hline Tumor localization & Extrahepatic/intrahepatic & $35 / 24$ & $1.772(0.677-4.638)$ & 0.244 & & n.s \\
\hline Operative tme & $\geq 427 /<427 \mathrm{~min}$ & $47 / 12$ & $2.869(0.962-8.561)$ & 0.059 & & n.s \\
\hline Intraoperative blood loss & $\geq 845 /<845 \mathrm{ml}$ & $30 / 29$ & $2.664(1.001-7.091)$ & 0.049 & $1.001(1.000-1.002)$ & 0.001 \\
\hline Serum CEA & $\geq 3.25 /<3.25 \mathrm{U} / \mathrm{ml}$ & $27 / 32$ & $2.846(1.094-7.406)$ & 0.032 & $6.051(1.484-24.669)$ & 0.012 \\
\hline GPS & $1,2 / 0$ & $11 / 48$ & $2.943(0.787-11.01)$ & 0.109 & & n.s \\
\hline NLR & $\geq 5.41 /<5.41$ & $5 / 54$ & $1.427(0.542-3.760)$ & 0.471 & & n.s \\
\hline PLR & $\geq 134 /<134$ & $38 / 21$ & $1.495(0.559-3.996)$ & 0.423 & & n.s \\
\hline PNI & $\geq 43 /<43$ & $42 / 17$ & $0.282(0.092-0.858)$ & 0.026 & & n.s \\
\hline CAR & $\geq 0.061 /<0.061$ & $31 / 28$ & $2.836(1.070-7.516)$ & 0.036 & & n.s \\
\hline PAR & $\geq 72.6 \times 10^{3 /<72.6 \times 10^{3}}$ & $16 / 43$ & $3.534(1.160-10.77)$ & 0.026 & $6.232(1.283-30.279)$ & 0.023 \\
\hline
\end{tabular}

CI: Confidence interval; extrahepatic: perihilar and distal bile duct carcinoma; intrahepatic: intrahepatic cholangiocarcinoma, cholangiocellular carcinoma, combined hepatocellular and cholangiocarcinoma; CEA: carcinoembryonic antigen; GPS: Glasgow prognostic score; HR: hazard ratio; NLR: neutrophil to lymphocyte ratio; n.s.: not significant; PLR: platelet to lymphocyte ratio; PNI: prognostic nutritional index; CAR: C-reactive protein to albumin ratio; PAR: platelet to albumin ratio.

of poor patient survival consisted of tumor stage $(p=0.0038)$, tumor differentiation $(p=0.002)$, nodal involvement $(p=0.029)$, intraoperative blood loss $(p=0.049)$, serum CEA $(p=0.032)$, PNI $(p=0.026)$, CAR $(p=0.036)$, and PAR $(p=0.026)$. In multivariate analysis, independent risk factors of poor patient survival consisted of poor tumor differentiation $(p=0.009)$, nodal involvement $(p=0.001)$, high intraoperative blood loss $(p=0.001)$, high serum CEA $(p=0.012)$, and high PAR $(p=0.023)$. ROC curves were calculated for survival status at the 3-year follow-up, and the AUC values were compared to assess cut-offs for each prognostic score as independent risk factors (Table III). The PAR had the highest AUC value $(0.709 \pm 0.08, p=0.002)$ compared to other prognostic scores as independent risk factors (Figure 1).

Relationship between clinical variables and DFS. The relationship between clinical variables and DFS are shown in Table IV. In univariate analysis, significant risk factors for cancer recurrence consisted of tumor stage $(p=0.0327)$, tumor differentiation $(p=0.004)$, serum CEA $(p=0.011)$, PNI $(p=0.016)$, and PAR $(p=0.020)$. In multivariate analysis, poor tumor differentiation $(p=0.009)$ and high PAR $(p=0.029)$ remained independent risk factors for cancer recurrence.

Univariate analysis of clinical variables in relation to PAR. The relationship between clinical variables and PAR are
Table III. Comparison of the area under the curve (AUC) between prognostic scores.

\begin{tabular}{lccc}
\hline Factor & AUC & $95 \%$ CI & $p$-Value \\
\hline Tumor differentiation & $0.678 \pm 0.08$ & $0.517-0.838$ & 0.002 \\
CEA & $0.674 \pm 0.37$ & $0.522-0.826$ & 0.032 \\
Nodal involvement & $0.604 \pm 0.08$ & $0.440-0.769$ & 0.029 \\
PNI & $0.652 \pm 0.08$ & $0.488-0.816$ & 0.026 \\
CAR & $0.633 \pm 0.08$ & $0.475-0.791$ & 0.036 \\
PAR & $0.709 \pm 0.08$ & $0.563-0.856$ & 0.002 \\
\hline
\end{tabular}

AUC: Area under the Receiver Operating Characteristics curve; CI: confidence interval; CEA: carcinoembryonic antigen; CAR: C-reactive protein to albumin ratio; PNI: prognostic nutritional index; PAR: platelet to albumin ratio.

shown in Table V. The group with a high PAR $\left(\geq 72.6 \times 10^{3}\right)$ group had advanced tumor stage $(p=0.048)$, higher PLR $(p=0.008)$ and lower PNI $(p=0.008)$.

Relationship between PAR and survival. In univariate analysis, the high PAR $\left(\geq 72.6 \times 10^{3}\right)$ group had a poor median OS (28.4 months, $p=0.0025)$ and DFS (23.1 months, $p=0.003$ ) (Figure 2); 3-year OS was $49.3 \%$ and $93.1 \%$ for the high and low PAR groups, respectively. 
Table IV. Univariate and multivariate analysis of clinicopathological variables in relation to disease-free survival.

\begin{tabular}{|c|c|c|c|c|c|c|}
\hline \multirow[b]{2}{*}{ Factor } & \multirow[b]{2}{*}{ Subgroup } & \multirow[b]{2}{*}{$\mathrm{N}$} & \multicolumn{2}{|c|}{ Univariate analysis } & \multicolumn{2}{|c|}{ Multivariate analysis } \\
\hline & & & HR $(95 \% \mathrm{CI})$ & $p$-Value & HR $(95 \% \mathrm{CI})$ & $p$-Value \\
\hline Tumor stage & $\mathrm{II}, \mathrm{III} / \mathrm{I}$ & $40 / 19$ & $2.913(1.092-7.771)$ & 0.0327 & & n.s \\
\hline Tumor size (mm) & $\geq 30.5 /<30.5 \mathrm{~mm}$ & $41 / 18$ & $1.822(0.636-5.221)$ & 0.264 & & n.s \\
\hline \multirow[t]{3}{*}{ Tumor differentiation } & Poor & 11 & $10.77(2.525-45.96)$ & 0.004 & $2.711(1.279-5.747)$ & 0.009 \\
\hline & Moderate & 30 & $1.652(0.466-5.857)$ & & & \\
\hline & Well (ref) & 18 & 1 & & & \\
\hline Nodal involvement & Positive/negative & $19 / 40$ & $2.887(0.972-8.569)$ & 0.056 & & \\
\hline Resection margin status & Positive/negative & $13 / 46$ & $1.258(0.416-3.808)$ & 0.684 & & $\mathrm{n} . \mathrm{s}$ \\
\hline Preoperative biliary drainage & Positive/negative & $16 / 43$ & $1.228(0.411-3.668)$ & 0.713 & & n.s \\
\hline Tumor localization & Extrahepatic/intrahepatic & $35 / 24$ & $1.534(0.580-4.056)$ & 0.389 & & n.s \\
\hline Operative tme & $\geq 427 /<427 \mathrm{~min}$ & $47 / 12$ & $1.335(0.856-2.133)$ & . & & n.s \\
\hline Intraoperative blood loss & $\geq 845 /<845 \mathrm{ml}$ & $30 / 29$ & $2.509(0.949-6.635)$ & 0.064 & & n.s \\
\hline Serum CEA & $\geq 3.25 /<3.25 \mathrm{U} / \mathrm{ml}$ & $27 / 32$ & $3.510(1.327-9.288)$ & 0.011 & & n.s \\
\hline GPS & $1,2 / 0$ & $11 / 48$ & $3.846(0.957-15.46)$ & 0.058 & & $\mathrm{n} . \mathrm{s}$ \\
\hline NLR & $\geq 5.41 /<5.41$ & $5 / 54$ & $1.576(0.584-4.254)$ & 0.369 & & n.s \\
\hline PLR & $\geq 134 /<134$ & $38 / 21$ & $1.709(0.626-4.667)$ & 0.295 & & n.s \\
\hline PNI & $\geq 43 /<43$ & $42 / 17$ & $0.247(0.079-0.771)$ & 0.016 & & $\mathrm{n} . \mathrm{s}$ \\
\hline CAR & $\geq 0.061 /<0.061$ & $31 / 28$ & $2.612(0.991-6.885)$ & 0.052 & & n.s \\
\hline PAR & $\geq 72.6 \times 10^{3} /<72.6 \times 10^{3}$ & $16 / 43$ & $3.789(1.227-11.70)$ & 0.020 & $4.422(1.168-16.732)$ & 0.029 \\
\hline
\end{tabular}

CI: Confidence interval; extrahepatic: perihilar and distal bile duct carcinoma; intrahepatic: intrahepatic cholangiocarcinoma, cholangiocellular carcinoma, combined hepatocellular and cholangiocarcinoma; CEA: carcinoembryonic antigen; GPS: Glasgow prognostic score; HR: hazard ratio; n.s.: not significant; NLR: neutrophil to lymphocyte ratio; PLR: platelet to lymphocyte ratio; PNI: prognostic nutritional index; CAR: C-reactive protein to albumin ratio; PAR: platelet to albumin ratio.

Table V. Univariate analysis of clinical variables in relation to preoperative platelet-to-albumin ratio (PAR).

\begin{tabular}{|c|c|c|c|c|}
\hline \multirow[t]{2}{*}{ Factor } & & \multicolumn{2}{|c|}{ PAR } & \multirow{2}{*}{$\begin{array}{c}p \text {-Value } \\
\text { (univariate) }\end{array}$} \\
\hline & & $<72.6 \times 10^{3}(n=43)$ & $\geq 72.6 \times 10^{3}(\mathrm{n}=16)$ & \\
\hline Tumor stage & $\mathrm{I}, \mathrm{II} / \mathrm{III}$ & $17 / 26$ & $2 / 14$ & 0.048 \\
\hline Tumor size & $<30.5 / \geq 30.5 \mathrm{~mm}$ & $13 / 30$ & $5 / 12$ & 0.940 \\
\hline Tumor differentiation & Well/moderate/poor & $13 / 23 / 7$ & $5 / 7 / 4$ & 0.706 \\
\hline Nodal involvement & Negative/positive & $30 / 13$ & $10 / 6$ & 0.595 \\
\hline Resection margin status & Negative/positive & $34 / 9$ & $12 / 4$ & 0.737 \\
\hline Preoperative biliary drainage & Negative/positive & $32 / 11$ & $11 / 5$ & 0.663 \\
\hline Operation & PD/HRx & $20 / 23$ & $4 / 12$ & 0.135 \\
\hline Operative time & $<427 / \geq 427 \mathrm{~min}$ & $11 / 32$ & $1 / 15$ & 0.101 \\
\hline Intraoperative blood loss & $<845 \mathrm{ml} / \geq 845 \mathrm{ml}$ & $23 / 20$ & $6 / 10$ & 0.275 \\
\hline Serum CEA & $<3.25 / \geq 3.25 \mathrm{U} / \mathrm{ml}$ & $24 / 19$ & $8 / 8$ & 0.690 \\
\hline GPS & $0 / 1,2$ & $36 / 7$ & $12 / 4$ & 0.444 \\
\hline NLR & $<5.41 / \geq 5.41$ & $28 / 15$ & $7 / 9$ & 0.137 \\
\hline PLR & $<134 / \geq 134$ & $32 / 11$ & $6 / 10$ & 0.008 \\
\hline PNI & $\geq 43 /<43$ & $7 / 36$ & $8 / 8$ & 0.008 \\
\hline CAR & $>0.061 / \geq 0.061$ & $26 / 17$ & $6 / 10$ & 0.115 \\
\hline
\end{tabular}

PD: Pancreaticoduodenectomy; HRx: hepatic resection; CEA: carcinoembryonic antigen; GPS: Glasgow prognostic score; NLR: neutrophil to lymphocyte ratio; PLR: platelet to lymphocyte ratio; PNI: prognostic nutritional index; CAR: C-reactive protein to albumin ratio; PAR: platelet to albumin ratio.

\section{Discussion}

The correlation between inflammation and each stage of cancer progression, such as carcinogenesis, promotion, progression and metastasis, has been reported $(18,19)$. In addition, several reports demonstrate a relationship between postoperative prognosis and systemic inflammation-based prognostic score such as GPS, modified GPS (20), NLR, PLR, PNI, and CAR. However, the relationships between these prognostic scores and therapeutic outcomes in cholangiocarcinoma have not been clarified. 

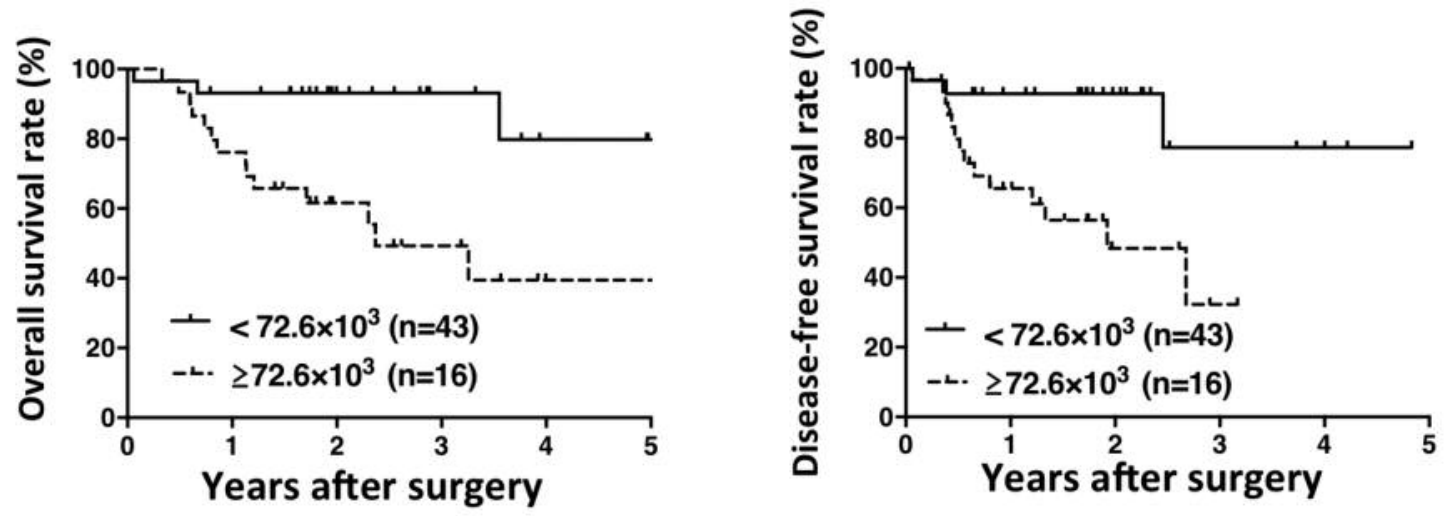

Figure 2. The relationship between platelet-to-albumin ratio $(P A R)$ and survival. The group with a high PAR $\left(\geq 72.6 \times 10^{3}\right)$ had a significantly poorer median overall survival (28.4 months, $p=0.0025)$ and disease-free survival (23.1 months, $p=0.003)$.

Some reports demonstrated that thrombocytosis, defined as a platelet count over $300-450 \times 10^{6} / 1$, was the significant risk factor in colorectal cancer (21-23). The most commonly expressed reasons for this correlation were induction of inflammation and protection from immune system surveillance (24). Platelets contain several types of cytokines that induce systemic inflammation such as interleukin 6 (IL6), transforming growth factor- $\beta$ (TGF- $\beta$ ), nuclear factor-kappa $\mathrm{B}$, and platelet-derived growth factor (PDGF) (25). These cytokines increase with thrombocytosis and affect cancer progression (19). Specifically, IL6 plays an important role in carcinogenesis and cancer progression (26); these severely worsen a patient's prognosis. In addition, PDGF activates cancer-associated fibroblasts, which play an active role in cell proliferation, production of anti-apoptotic signals and tumor progression (27, 28). Cancer-associated fibroblasts are highly prevalent in cholangiocarcinoma, therefore, a high serum platelet count would seem to affect the condition of patients with cholangiocarcinoma (29).

The relationship between hypoalbuminemia and short life expectancy in patients with cancer has also been broadly recognized (30). Hypoalbuminemia is seen in patients with malnutrition and cachexia, increasing the risk of anticancer agent-induced toxicity. Furthermore, systemic inflammation suppresses albumin synthesis in hepatocytes by the production of cytokines. Hypoalbuminemia is also associated with failure of various immune system components (31) and helps tumor cells to progress.

In summary, because PAR reflects both the platelet count and serum albumin level, it seems to be a useful index for patients with cholangiocarcinoma after primary resection. PAR also has other advantages. The platelet count and serum albumin level are usually examined at every institution before surgery without excessive cost. Furthermore, PAR can easily be calculated dividing the platelet count by the serum albumin level. Limitations of the current study include its retrospective design with potential biases. By accumulating data prospectively, the benefit of neoadjuvant chemotherapy before resection for cholangiocarcinoma may be clarified by PAR.

\section{Conflicts of Interest}

No Author has any conflicts of interest to declare in regard to the current study.

\section{References}

1 Blechacz B and Gores GJ: Cholangiocarcinoma: advances in pathogenesis, diagnosis, and treatment. Hepatology 48: 308-321, 2008.

2 Cunningham SC, Choti MA, Bellavance EC and Pawlik TM: Palliation of hepatic tumors. Surg Oncol 16: 277-291, 2007.

3 Valle JW: Advances in the treatment of metastatic or unresectable biliary tract cancer. Ann Oncol 21(Suppl 7): vii345$348,2010$.

4 D'Angelica MI, Jarnagin WR and Blumgart LH: Resectable hilar cholangiocarcinoma: surgical treatment and long-term outcome. Surg Today 34: 885-890, 2004.

5 Le Roy B, Gelli M, Pittau G, Allard MA, Pereira B, Serji B, Vibert E, Castaing D, Adam R, Cherqui D and Sa Cunha A: Neoadjuvant chemotherapy for initially unresectable intrahepatic cholangiocarcinoma. Br J Surg, 2017. doi: 10.1002/bjs.10641. [Epub ahead of print]

6 Reames BN, Bagante F, Ejaz A, Spolverato G, Ruzzenente A, Weiss M, Alexandrescu S, Marques HP, Aldrighetti L, Maithel SK, Pulitano C, Bauer TW, Shen F, Poultsides GA, Soubrane O, Martel G, Koerkamp BG, Guglielmi A, Itaru E and Pawlik TM: Impact of adjuvant chemotherapy on survival in patients with intrahepatic cholangiocarcinoma: a multi-institutional analysis. HPB (Oxford) 19: 901-909, 2017. 
7 Pang Q, Zhang JY, Xu XS, Song SD, Qu K, Chen W, Zhou YY, Miao RC, Liu SS, Dong YF and Liu C: Significance of platelet count and platelet-based models for hepatocellular carcinoma recurrence. World J Gastroenterol 21: 5607-5621, 2015.

8 Prokopowicz G, Zyczkowski M, Nowakowski K, Bogacki R, Bryniarski P and Paradysz A: Basic parameters of blood count as prognostic factors for renal cell carcinoma. Biomed Res Int 2016: 8687575, 2016.

9 Ge X, Dai X, Ding C, Tian H, Yang J, Gong J, Zhu W, Li N and Li J: Early postoperative decrease of serum albumin predicts surgical outcome in patients undergoing colorectal resection. Dis Colon Rectum 60: 326-334, 2017.

10 Han WX, Chen ZM, Wei ZJ and Xu AM: Preoperative prealbumin predicts prognosis of patients after gastrectomy for adenocarcinoma of esophagogastric junction. World J Surg Oncol 14: 279, 2016.

11 Sobin LH and Compton CC: TNM seventh edition: What's new, what's changed: Communication from the International Union Against Cancer and the American Joint Committee on Cancer. Cancer 116: 5336-5339, 2010.

12 Moriwaki T, Ishige K, Araki M, Yoshida S, Nishi M, Sato M, Yamada T, Yamamoto Y, Ozeki M, Ishida H, Yamaguchi T, Matsuda K, Murashita T, Abei M and Hyodo I: Glasgow Prognostic Score predicts poor prognosis among advanced biliary tract cancer patients with good performance status. Med Oncol 31: 287, 2014.

13 Chen Q, Dai Z, Yin D, Yang LX, Wang Z, Xiao YS, Fan J and Zhou J: Negative impact of preoperative platelet-lymphocyte ratio on outcome after hepatic resection for intrahepatic cholangiocarcinoma. Medicine (Baltimore) 94: e574, 2015.

14 Shirai Y, Shiba H, Sakamoto T, Horiuchi T, Haruki K, Fujiwara Y, Futagawa Y, Ohashi T and Yanaga K: Preoperative platelet to lymphocyte ratio predicts outcome of patients with pancreatic ductal adenocarcinoma after pancreatic resection. Surgery 158 : 360-365, 2015.

15 Zhang C, Wang H, Ning Z, Xu L, Zhuang L, Wang P and Meng $\mathrm{Z}$ : Prognostic nutritional index serves as a predictive marker of survival and associates with systemic inflammatory response in metastatic intrahepatic cholangiocarcinoma. Onco Targets Ther 9: 6417-6423, 2016.

16 Haruki K, Shiba H, Shirai Y, Horiuchi T, Iwase R, Fujiwara Y, Furukawa $\mathrm{K}$, Misawa $\mathrm{T}$ and Yanaga $\mathrm{K}$ : The $\mathrm{C}$-reactive protein to albumin ratio predicts long-term outcomes in patients with pancreatic cancer after pancreatic resection. World J Surg 40: 2254-2260, 2016.

17 Shirai Y, Shiba H, Haruki K, Horiuchi T, Saito N, Fujiwara Y, Sakamoto T, Uwagawa T and Yanaga K: Preoperative platelet to albumin ratio predicts prognosis of patients with pancreatic ductal adenocarcinoma after pancreatic resection. Anticancer Res 37: 787-793, 2017.

18 Han WX, Chen ZM, Wei ZJ and Xu AM: Preoperative prealbumin predicts prognosis of patients after gastrectomy for adenocarcinoma of esophagogastric junction. World J Surg Oncol 14: 279, 2016
19 Mantovani A, Allavena P, Sica A and Balkwill F: Cancer-related inflammation. Nature 454: 436-444, 2008.

20 Okuno M, Ebata T, Yokoyama Y, Igami T, Sugawara G, Mizuno $\mathrm{T}$, Yamaguchi $\mathrm{J}$ and Nagino M: Evaluation of inflammation-based prognostic scores in patients undergoing hepatobiliary resection for perihilar cholangiocarcinoma. J Gastroenterol 51: 153-161, 2016.

21 Cheng J, Zeng Z, Ye Q, Zhang Y, Yan R, Liang C, Wang J, Li $\mathrm{M}$ and Yi M: The association of pretreatment thrombocytosis with prognosis and clinicopathological significance in cervical cancer: a systematic review and meta-analysis. Oncotarget 8 : 24327-24336, 2017.

22 Ishizuka M, Nagata H, Takagi K, Iwasaki Y and Kubota K: Preoperative thrombocytosis is associated with survival after surgery for colorectal cancer. J Surg Oncol 106: 887-891, 2012.

23 Nyasavajjala SM, Runau F, Datta S, Annette H, Shaw AG and Lund JN: Is there a role for pre-operative thrombocytosis in the management of colorectal cancer? Int J Surg 8: 436-438, 2010.

24 Steele M and Voutsadakis IA: Pre-treatment platelet counts as a prognostic and predictive factor in stage II and III rectal adenocarcinoma. World J Gastrointest Oncol 9: 42-49, 2017.

25 Mussano F, Genova T, Munaron L, Petrillo S, Erovigni F and Carossa S: Cytokine, chemokine, and growth factor profile of platelet-rich plasma. Platelets 27: 467-471, 2016.

26 Sido A, Radhakrishnan S, Kim SW, Eriksson E, Shen F, Li Q, Bhat V, Reddivari L and Vanamala JKP: A food-based approach that targets interleukin-6, a key regulator of chronic intestinal inflammation and colon carcinogenesis. J Nutr Biochem 43: 11$17,2017$.

27 Anderberg C and Pietras K: On the origin of cancer-associated fibroblasts. Cell Cycle 8: 1461-1462, 2009.

28 Berasain C and Avila MA: Platelet-derived growth factor D: a new player in the complex cross-talk between cholangiocarcinoma cells and cancer-associated fibroblasts. Hepatology 58: 853-855, 2013.

29 Cadamuro M, Nardo G, Indraccolo S, Dall'olmo L, Sambado L, Moserle L, Franceschet I, Colledan M, Massani M, Stecca T, Bassi N, Morton S, Spirli C, Fiorotto R, Fabris L and Strazzabosco M: Platelet-derived growth factor-D and Rho GTPases regulate recruitment of cancer-associated fibroblasts in cholangiocarcinoma. Hepatology 58: 1042-1053, 2013.

30 Gupta D and Lis CG: Pretreatment serum albumin as a predictor of cancer survival: a systematic review of the epidemiological literature. Nutr J 9: 69, 2010.

31 Lin MY, Liu WY, Tolan AM, Aboulian A, Petrie BA and Stabile BE: Preoperative serum albumin but not prealbumin is an excellent predictor of postoperative complications and mortality in patients with gastrointestinal cancer. Am Surg 77: 1286-1289, 2011.

Received October 31, 2017

Revised November 22, 2017

Accepted November 28, 2017 